Declared, A. Winstanley: None Declared, M. Rodriguez-Justo: None Declared, M. Novelli: None Declared, M. Banks: None Declared, S. Bown: None Declared, L. Lovat Grant/Research Support from: DUSA Pharmaceuticals.

Keywords Barrett's oesophagus, High grade dysplasia, Photodynamic therapy.

\section{PWE-102 A RANDOMISED CONTROLLED TRIAL OF ALA VERSUS PHOTOFRIN PDT FOR HIGH GRADE DYSPLASIA IN BARRETT'S OESOPHAGUS}

\author{
doi:10.1136/gut.2011.239301.365
}

J M Dunn, ${ }^{1, *}$ G D Mackenzie, ${ }^{1}$ S Thorpe, ${ }^{1}$ M Austwick, ${ }^{1}$ C A Mosse, ${ }^{1}$ D Oukrif, ${ }^{2}$ A Winstanley, ${ }^{2}$ M Rodriguez-Justo, ${ }^{2}$ M R Novelli, ${ }^{2}$ M R Banks, ${ }^{3}$ S G Bown, ${ }^{1}$ L B Lovat $1,3{ }^{1}$ National Medical Laser Centre, University College London, London, UK; ${ }^{2}$ Histopathology, University College London, London, UK; ${ }^{3}$ Gastroenterology, University College London Hospitals NHS Trust, London, UK

Introduction Sodium porfimer photodynamic therapy (spPDT) is a licensed minimally invasive treatment for Barrett's Oesophagus (BO) with high grade dysplasia (HGD). Complete reversal (CR)-HGD is $50 \%$ at 5 years. sp-PDT is associated with a significant risk of strictures and prolonged photosensitivity up to 3 months. 5 aminolaevulinic acid (ALA) is an alternative treatment with 36 h photosensitivity. Non-randomised data suggests $85 \%$ CR-HGD and low risk of side effects. The aim of this study was to compare the side effect profile between the drugs and outcomes of therapy.

Methods Single centre randomised controlled trial. Presence of HGD was confirmed on two occasions by two specialist GI pathologists. All patients had Endoscopic Ultrasound and endoscopic resection of visible lesions prior to PDT. Stratification was by length of $\mathrm{BO}(<>6 \mathrm{~cm})$ and extent of dysplasia. Standard protocols for ALA and sp-PDT were followed. Endoscopic follow-up with $2 \mathrm{~cm}$ four-quadrant biopsy was at 6 weeks, 4 months, then annually. All adverse event data were collected.

Results Sixty-four patients were randomised, 34 ALA and 30 sp-PDT. Median follow-up is currently 23 months. Strictures and skin photosensitivity were significantly more common with sp-PDT than ALA-PDT (33\% vs $9 \%$ and $43 \%$ vs $6 \%$ respectively) $p<0.05$. Preliminary outcome data show CR-HGD in $17 / 34$ (50\%) with ALA-PDT and $12 / 30(40 \%)$ with sp-PDT. Overall cancer incidence was $10 \%$, no significant difference between groups. For $\mathrm{BO} \leq 6 \mathrm{~cm}$, using log-rank analysis CR-HGD was more likely with ALA-PDT (78\%) than sp-PDT $(31 \%), \mathrm{X}^{2}=4.34, \mathrm{p}=0.037$. For $\mathrm{BO}$ length $>6 \mathrm{~cm}$ there was no significant difference. Rate of buried glands was significantly higher post PDT ( $48 \%$ ) than pre PDT $(20 \%)$, no significant difference between groups.

Conclusion ALA-PDT has a better risk profile than sp-PDT. In patients with $B O$ length $\leq 6 \mathrm{~cm}$, preliminary results show ALA-PDT is associated with significantly higher CR-HGD. In longer segments of BO neither PDT drug was sufficiently efficacious to warrant use.

Competing interests J. Dunn: None Declared, G. Mackenzie: None Declared, S. Thorpe: None Declared, M. Austwick: None Declared, C. Mosse: None Declared, D. Oukrif: None 